\title{
Prediction of Water Consumption in Hospitals Based on a Modified Grey GM $(0,1 \mid \sin )$ Model of Oscillation Sequence: The Example of Wuhan City
}

\author{
Han Zhou, Jiejun Huang, Yanbin Yuan, and Biao Tang \\ School of Resource and Environmental Engineering, Wuhan University of Technology, Wuhan 430070, China \\ Correspondence should be addressed to Jiejun Huang; hjj@whut.edu.cn
}

Received 7 March 2014; Accepted 13 June 2014; Published 3 July 2014

Academic Editor: Hector Pomares

Copyright (c) 2014 Han Zhou et al. This is an open access article distributed under the Creative Commons Attribution License, which permits unrestricted use, distribution, and reproduction in any medium, provided the original work is properly cited.

Water shortage is one of the main factors limiting urban construction and development. Scientific forecasting of water consumption is an important approach for the rational allocation of water resources. Taking the hospitals in Wuhan City as an example and basing the analysis on the characteristics of actual water consumption, we proposed a modified grey GM $(0,1 \mid \sin )$ model of oscillation sequence. Using the grey theory, the variable weight-strengthening buffer operator (VWSBO) was introduced into this model to weaken the interference of the disturbance term on the data sequence. The actual quarterly total water consumption data for hospitals in Wuhan City during the period from 2010 to 2012 were used to verify the effectiveness and practicality of this modified grey GM $(0,1 \mid \sin )$ model in predicting water consumption. In terms of the model's fitting performance, the mean absolute percentage error (MAPE) of the modified model was 3.77\%, indicating a higher prediction accuracy than the traditional grey GM $(0,1 \mid \sin )$ model of oscillation sequences. Therefore, the modified grey GM $(0,1 \mid \sin )$ model we established in this study can provide a scientific reference for administrative departments to forecast water consumption.

\section{Introduction}

Water is one of the most important natural resources for productive activities in human society. The total water resources in China are abundant. However, the per capita amount of water is low, and pollution is a serious concern, leading to a large imbalance between water supply and demand that has greatly hindered the development of the social economy. To solve the problem of water shortages, the rational allocation of water resources is an important goal. Predicting water demand can provide a reference to plan for the rational allocation and management of water resources, and it has important practical significance in the conservation and effective use of water.

Currently, there are a variety of methods and models for forecasting water consumption, including regression analysis [1], the exponential smoothing method [2], and backpropagation (BP) of an artificial neural network [3,4]. The grey system theory was proposed by a Chinese scholar, Professor Deng Julong, in 1982. Due to its unique advantages in studying problems with insufficient data, poor information, and uncertainty, this theory has been widely applied in many fields such as industry [5], electric power [6], agriculture [7], geology $[8]$, healthcare $[9,10]$, economy $[11,12]$, education [13], and municipal water conservation [14-16]. Although the classic GM $(1,1)$ model can achieve relatively satisfactory performance, its application is limited in analyzing data that have minimal discreteness and show only a monotonic changing trend; thus, this classic model cannot be used to analyze the regularity of fluctuations in periodic-oscillation sequences. The grey GM $(0,1 \mid \sin )$ model of oscillation sequences [17] integrates the two mathematical methods of the grey system and Fourier transformation to overcome the limitations of the classic GM $(1,1)$ prediction model. This grey GM $(0,1 \mid \sin )$ model of oscillation sequences can effectively identify the impact of an internal disturbance factor on the development of a system and analyze the regularity of the fluctuations in the system, as validated by the experiments using measured data for short-term traffic flow. However, the grey GM (0, 1|sin) model of oscillation sequences does not take into 
account the distortion of the system behavioral data caused by the interference of certain disturbance items within the system itself. In the forecasting process, disturbances always invalidate the forecasting model; however, the invalidation arises from the distortion of the system's behavioral data caused by disturbances rather than the selection of model [18]. Liu investigated the characteristics and axiom system of buffer operators and constructed two types of buffer operators [19]. In recent years, buffer operators have been widely applied in various fields; however, traditional buffer operators cannot achieve the fine-tuning of function strength, resulting in a buffer that is either too strong or too weak. To solve this problem, previous studies [20, 21] introduced a type of variable-weight buffer operator and investigated the relationship between the adjusting degree of a buffer operator and the variable weight. The superiority and effectiveness of variable-weight buffer operators have been verified using real-life examples.

Taking into account the multiple characteristics of quarterly water consumption data in hospitals of Wuhan City such as uncertainty, periodic oscillations, and uneven growth, we conducted a qualitative analysis of the changing trends in water consumption and a quantitative analysis of the function of the variable-weight-strengthening buffer operator (VWSBO). By introducing the VWSBO to fine-tune the disturbed data sequence, we modified the previous grey GM $(0,1 \mid \sin )$ model of oscillation sequences to improve its performance in simulating the characteristics of the water consumption in the hospitals of Wuhan City to achieve higher prediction accuracy.

\section{Materials and Methods}

2.1. Grey GM $(0,1 \mid \sin )$ Model of Oscillation Sequences. The data sequence of the quarterly water consumption in hospitals shows obvious oscillation and periodicity similar to the annual growth rate. The annulus development coefficient fluctuates above and below a certain value, showing obvious features of a sine or cosine curve; thus, sine and cosine curves were superpositioned to fit the fluctuation of the data.

The modeling procedure of the grey $\operatorname{GM}(0,1 \mid \sin )$ model of oscillation sequences is as follows [17].

(1) Given an original nonnegative sequence

$$
x^{(0)}=(x(1), x(2), \ldots, x(n)) \quad n \in N^{+},
$$

the annulus development coefficient is

$$
a(k)=\ln \frac{x(k)}{x(k-1)} .
$$

(2) The whitening equation of the model is

$$
\frac{d x^{(0)}}{d t}+a \sin (\omega t-p) x^{(0)}=b x^{(0)},
$$

where the parameter $b$ of the oscillation grey model roughly determines the trend of the sequence. When $b>0$, the trend is ascending, and when $b<0$, the trend is descending. The parameter $\omega$ describes the ascending or descending variation of the sequence and determines its oscillation cycle. The parameter $a$ determines the amplitude of the oscillation, $t$ denotes time variable, and $p$ denotes phase.

(3) When $a \sin (\omega t-p)=a_{1} \sin (\omega t)+a_{2} \cos (\omega t)$, the differential equation (3) is solved to be

$$
\ln x^{(0)}=\frac{a_{1} \cos (\omega t)-a_{2} \sin (\omega t)}{\omega}+b t+c .
$$

Thus,

$$
x^{(0)}(t)=C \exp \left(\frac{a_{1} \cos (\omega t)-a_{2} \sin (\omega t)}{\omega}+b t\right) ;
$$

an initial value of $x^{(0)}(1)$ is substituted to obtain $C=$ $x^{(0)}(1) \exp \left(\left(-a_{1} \cos (\omega)+a_{2} \sin (\omega)\right) / \omega-b\right)$; the resulting timecorresponding sequence of the $\operatorname{GM}(0,1 \mid \sin )$ model is

$$
\begin{gathered}
x^{(0)}(t)=x^{(0)}(1) \exp \left(\frac{a_{1} \cos (\omega t)-a_{2} \sin (\omega t)}{\omega}+b(t-1)\right. \\
\left.+\frac{-a_{1} \cos (\omega)+a_{2} \sin (\omega)}{\omega}\right) .
\end{gathered}
$$

(4) Fourier transform is applied to solve the periodic parameters.

The annulus development coefficient $a(k)$ fluctuates above and below the value of 0 in a sine or cosine manner, so a finite discrete Fourier transform is conducted for $a(k)$, to seek $m$ frequencies with the greater amplitude:

$$
F(m \Omega)=\sum_{k=0}^{n-1} a(k) e^{-j m \Omega k}, \quad m=0,1,2, \ldots, n-1 .
$$

The equation can be reorganized as $F(m \Omega)=R_{m}+j I_{m}$,

$$
\begin{aligned}
R_{m}= & a(0) \cos \left(\frac{2 \pi}{n} m \cdot 0\right)+a(1) \cos \left(\frac{2 \pi}{n} m \cdot 1\right) \\
& +\cdots+a(n-1) \cos \left(\frac{2 \pi}{n} m \cdot(n-1)\right), \\
I_{m}=- & {\left[a(0) \sin \left(\frac{2 \pi}{n} m \cdot 0\right)+a(1) \sin \left(\frac{2 \pi}{n} m \cdot 1\right)\right.} \\
& \left.+\cdots+a(n-1) \sin \left(\frac{2 \pi}{n} m \cdot(n-1)\right)\right] .
\end{aligned}
$$

The Fourier transform coefficient $F(m \Omega)$ can be obtained from (7). The frequency corresponding to the large 
$|F(m \Omega)|^{2}=R_{m}{ }^{2}+I_{m}{ }^{2}$ serves as the whitening value of the grey frequency.

(5) The parameters of the GM $(0,1 \mid \sin )$ model are solved with the least squares method.

$$
\begin{gathered}
\mathbf{Y}=\left[\begin{array}{c}
\ln \frac{x(2)}{x(1)} \\
\ln \frac{x(3)}{x(2)} \\
\vdots \\
\ln \frac{x(n)}{x(n-1)}
\end{array}\right], \\
\mathbf{B}=\left[\begin{array}{c}
\frac{\cos (2 \omega)-\cos (\omega)}{\omega} \\
\frac{\cos (3 \omega)-\cos (2 \omega)}{\omega} \\
\vdots \\
\frac{\cos (n \omega)-\cos ((n-1) \omega)}{\omega}
\end{array}\right.
\end{gathered}
$$

where $\mathbf{B}$ is a matrix of the independent variables, $\mathbf{Y}$ is the vector of a dependent variable, and $\mathbf{u}$ is the vector of a coefficient.

2.2. Introducing the VWSBO to Improve the Oscillation Grey Model. Before obtaining the equation for trend prediction, the growth trend and the oscillating trend of the sequence need to be strengthened. With the introduction of the VWSBO, a strengthened buffer sequence can be obtained:

$$
x^{(0)} D=(x(1) d, x(2) d, \ldots, x(n) d) \quad n \in N^{+},
$$

where

$$
x(k) d=x(k)\left(\frac{x(k)}{\lambda \min \left(x^{(0)}\right)+(1-\lambda) x(k)}\right)^{\gamma},
$$

$\lambda$ is the variable weight, and $0<\lambda<1$.

Because $x(k)>\lambda \min \left(x^{(0)}\right)+(1-\lambda) x(k)$, when $\gamma>0$, whether $x^{(0)}$ is a monotonic ascending sequence, a monotonic descending sequence, or an oscillation sequence, $D$ is the strengthening buffer operator.

2.3. The Function Strength of the VWSBO. Let $r(k)$ be the average rate of the change from $x(k)$ to $\min \left(x^{(0)}\right)$ in the data sequence $x^{(0)}$; thus, $D$ is the VWSBO on $x^{(0)}$. After proceeding $x^{(0)}$ with the VWSBO $D$, the resulting data sequence is presented in (10), and thus

$$
\delta(k)=\left|\frac{r(k)-r(k) d}{r(k)}\right| .
$$

It is the adjusting degree of the VWSBO $D$ at point $k$ that indicates that $\delta(k)$ is the functioning-strength value of the VWSBO.
The response sequence parameter was estimated based on the whitening equation with the minimum variance of the annulus development coefficient: $\mathbf{u}=\left(\mathbf{B}^{\mathrm{T}} \mathbf{B}\right)^{-\mathbf{1}} \mathbf{B}^{\mathrm{T}} \mathbf{Y}$.

$\mathbf{B}, \mathbf{Y}$, and $\mathbf{u}$ can be obtained from (4):

$$
\mathbf{u}=\left[\begin{array}{lll}
a_{1} & a_{2} & b
\end{array}\right]^{T},
$$

$$
\left.\begin{array}{cc}
-\frac{\sin (2 \omega)-\sin (\omega)}{\omega} & 1 \\
-\frac{\sin (3 \omega)-\sin (2 \omega)}{\omega} & 1 \\
\vdots & \vdots \\
-\frac{\sin (n \omega)-\sin ((n-1) \omega)}{\omega} &
\end{array}\right] \text {, }
$$

According to (10) and (12),

$$
\begin{aligned}
r(k)-r(k) d= & \frac{\min \left(x^{(0)}\right)-x(k) d}{n-k+1}-\frac{\min \left(x^{(0)}\right)-x(k)}{n-k+1} \\
= & \frac{x(k)}{n-k+1} \\
& \times\left[1-\left(\frac{x(k)}{\lambda \min \left(x^{(0)}\right)+(1-\lambda) x(k)}\right)^{\gamma}\right] .
\end{aligned}
$$

Thus,

$$
\begin{aligned}
\delta(k)= & \left|\frac{r(k)-r(k) d}{r(k)}\right| \\
= & \frac{x(k)}{\min \left(x^{(0)}\right)-x(k)} \\
& \times\left[1-\left(\frac{x(k)}{\lambda \min \left(x^{(0)}\right)+(1-\lambda) x(k)}\right)^{\gamma}\right]>0,
\end{aligned}
$$

where $\min \left(x^{(0)}\right) \neq x(k), k=1,2, \ldots, n$.

Thus,

$$
\begin{aligned}
\frac{d \delta}{d \lambda}= & \gamma\left(\frac{x(k)}{\lambda \min \left(x^{(0)}\right)+(1-\lambda) x(k)}\right)^{\gamma+1}>0 \\
\frac{d \delta}{d \gamma}= & \frac{x(k)}{\min \left(x^{(0)}\right)-x(k)}\left(\frac{x(k)}{\lambda \min \left(x^{(0)}\right)+(1-\lambda) x(k)}\right)^{\gamma} \\
& \times \ln \left(\frac{x(k)}{\lambda \min \left(x^{(0)}\right)+(1-\lambda) x(k)}\right)>0 .
\end{aligned}
$$


This result shows that the adjusting degree $\delta(k)$ of the VWSBO $D$ on a sequence changes in the same direction as the variable weight $\lambda$ and the index $\gamma$.

The qualitative analysis was carried out for the changing trend of the quarterly water consumption in the hospitals, and the quantitative analysis was carried out for the function strength of the VWSBO. This study introduced a VWSBO to fine-tune the original system's behavioral data sequence and then constructed a modified grey GM $(0,1 \mid \sin )$ model of oscillation sequences following the procedure of Section 2.1, which can be applied in forecasting the water consumption and planning guidance for the hospitals of Wuhan City.

2.4. The Degree of Grey Incidence Analysis. According to the grey system theory, first we set the original sequence $x^{(0)}$ as reference, so the compared sequences are $X_{i}(k)=\left\{x_{i}(k) \mid\right.$ $k=1,2, \ldots, n\}(i=1,2, \ldots, N)$. Then, we initialize based on $x_{i}^{\prime}(k)=x_{i}(k) / x_{i}(1) ; x^{\prime(0)}(k)=x^{(0)}(k) / x^{\prime(0)}(1)$ in order to keep the data dimension consistent between the reference sequence and compared sequence. The maximum absolute difference $\Delta_{\max }$ and the minimum absolute difference $\Delta_{\min }$ can be obtained by using $\Delta_{i}=\left|x^{(0)}(k)-x_{i}(k)\right|$ to compute the difference sequence. Then the degree of grey incidence $\beta$ can be calculated by

$$
\beta\left(x^{(0)}, x_{i}\right)=\frac{1}{n} \sum_{k=1}^{n} \xi\left(x^{(0)}(k), x_{i}(k)\right),
$$

where

$$
\begin{aligned}
& \xi\left(x^{(0)}(k), x_{i}(k)\right) \\
& =\frac{\min _{i} \min _{k}\left|x^{(0)}(k)-x_{i}(k)\right|+\rho \max _{i} \max _{k}\left|x^{(0)}(k)-x_{i}(k)\right|}{\left|x^{(0)}(k)-x_{i}(k)\right|+\rho \max _{i} \max _{k}\left|x^{(0)}(k)-x_{i}(k)\right|} .
\end{aligned}
$$

In (17), $\xi\left(x^{(0)}(k), x_{i}(k)\right)$ is the grey incidence coefficient of sequences $x^{(0)}(k)$ and $x_{i}(k)$ at point $k . \rho$ denotes distinguished coefficient and $\rho \in[0,1]$. Generally, $\rho \leq 0.5463$ would be considered satisfactory for analysis. Empirically, when $\rho=0.5$ and the degree of grey incidence $\beta>0.6$, the model is considered to have good precision. A greater degree of grey incidence indicates less deviation of the compared sequence from the reference sequence, which means greater influence on the reference sequence.

\section{Results and Discussion}

3.1. Data Sources. To verify the superiority of the modified grey model of oscillation sequences after the introduction of the VWSBO, this study used the quarterly total water consumption data for nearly 75 hospitals in Wuhan City between 2010 and 2012 as an example. Water consumption data has high volatility and uncertainty, and it is difficult to sum up the regularity from the monthly data, so the paper have chosen quarterly data to lower the volatility as well as the influence caused by uncertainty. Combined with the fact that water consumption of hospital industry in Wuhan has
TABLE 1: The quarterly total water consumption by the hospitals in Wuhan City in 2010-2012 ( $\times 10^{4}$ tons).

\begin{tabular}{lcccc}
\hline Year\quarter & $\begin{array}{c}\text { The first } \\
\text { quarter }\end{array}$ & $\begin{array}{c}\text { The second } \\
\text { quarter }\end{array}$ & $\begin{array}{c}\text { The third } \\
\text { quarter }\end{array}$ & $\begin{array}{c}\text { The fourth } \\
\text { quarter }\end{array}$ \\
\hline 2010 & 264.3 & 327.1 & 317.6 & 286.2 \\
2011 & 292.2 & 300.5 & 324.4 & 300.1 \\
2012 & 360.0 & 328.5 & 368.9 & 307.6 \\
\hline
\end{tabular}

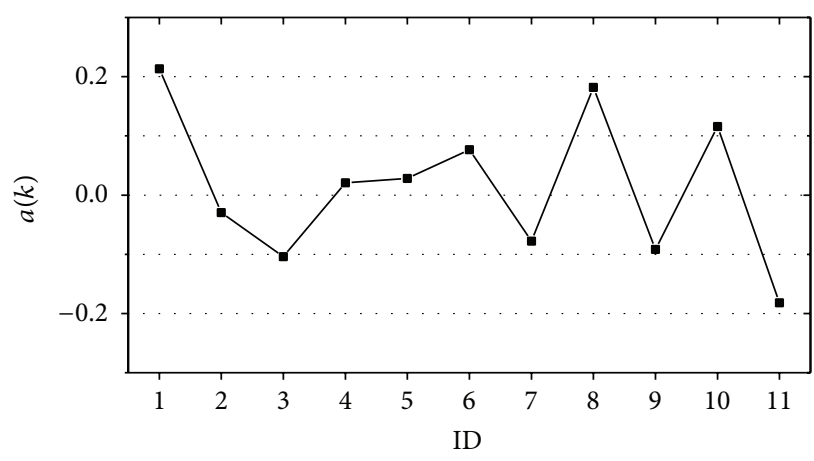

FIGURE 1: Line graph of the annulus development coefficient.

seasonality effect, the study decided to use quarterly data rather than monthly data. Then, the predicted results using the model before and after the modification were analyzed and compared.

The experimental data are shown in Table 1.

3.2. Calculation of the Annulus Development Coefficient. The raw data sequence of the quarterly water consumption is as follows.

Based on (2), the line graph of the annulus development coefficient $a(k)$ can be obtained, as shown in Figure 1 .

Figure 1 demonstrates that the annulus development coefficient $a(k)$ fluctuates above and below the value 0 in a sine or cosine curve; thus, the superposition of sine and cosine can be used to fit the fluctuation of the annulus development coefficient.

\subsection{The Modified Grey GM (0, 1|sin) Model of Oscillation} Sequences. The introduction of the VWSBO improved the grey GM $(0,1 \mid \sin )$ model of oscillation sequences based primarily on the analysis of the following two aspects.

The qualitative analysis of the development of hospitals in Wuhan City in recent years showed that, since 2009, while the number of hospitals increased slightly, the total number of hospital beds in Wuhan City increased at an average annual growth rate of $8.7 \%$ and the average number of hospitalizations increased at an annual rate of $15.2 \%$. With the rapid development of urban construction in Wuhan City, the development of hospitals in Wuhan City will continue at an accelerating rate, which will inevitably lead to an increase in water consumption. Although the implementation of watermanagement plans in Wuhan City has achieved initial success in recent years, the increase in water consumption due to the development of industry could not be offset. 


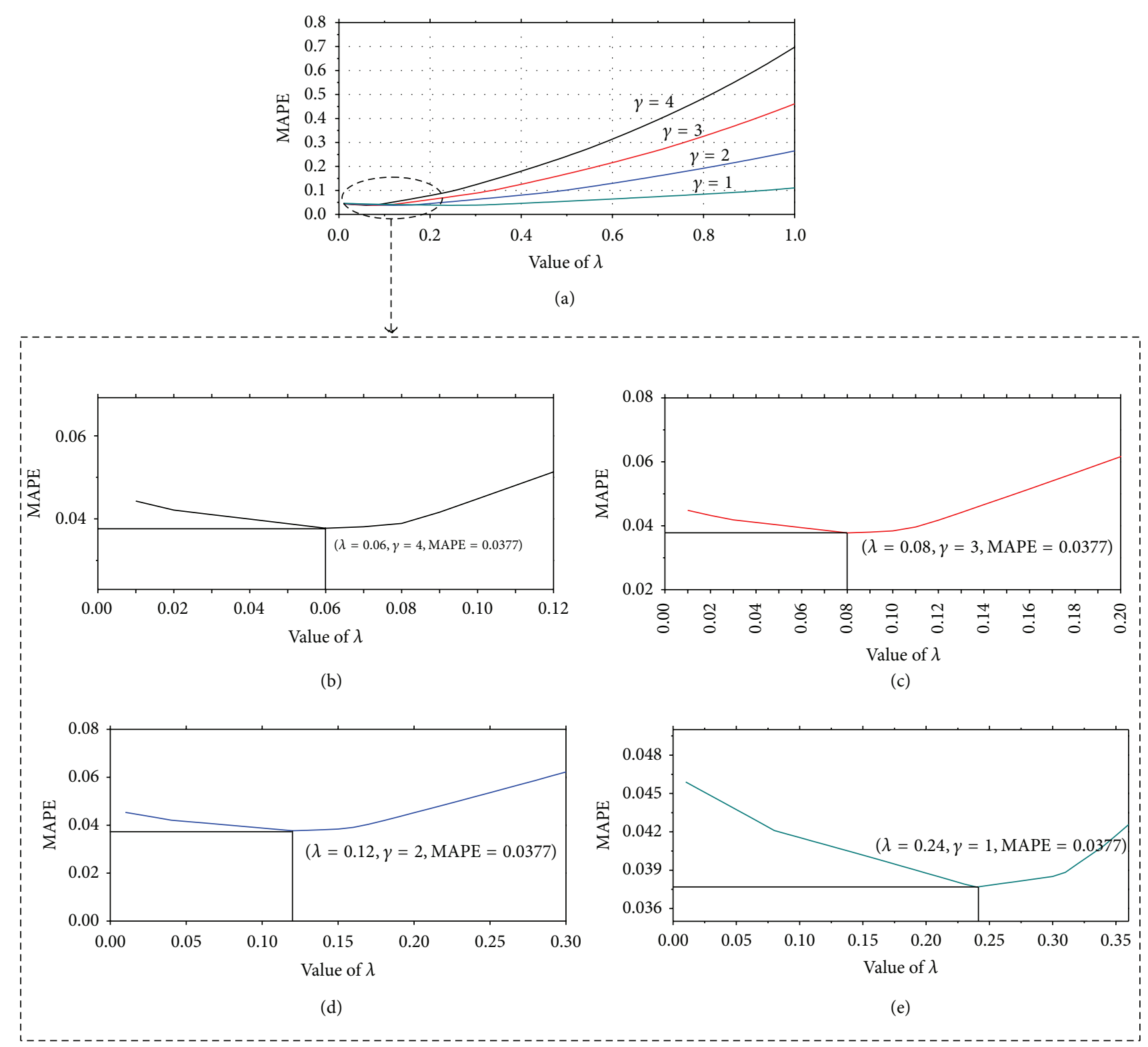

Figure 2: The trend of the function strength of the VWSBO with different indexes: (a) different indexes; (b) $\gamma=4, \lambda=0.06$; (c) $\gamma=3$, $\lambda=0.08$; (d) $\gamma=2, \lambda=0.12$; (e) $\gamma=1, \lambda=0.24$.

Quantitatively, this study analyzed the trend of the function strength of the VWSBO based on the criterion of error minimization under different circumstances. The results are shown in Figure 2.

Therefore, to accurately control the future water demand of the hospitals in Wuhan City, this study applied a strong function strength in the quarterly water consumption sequence with $\gamma=3$ and $\lambda=0.08$.

The data sequence after processing with the VWSBO is

$x D=(264.3,342.6,330.7,291.5,299.0,309.4,339.3,308.9$,

$384.0,344.4,395.2,318.2)$.
The sequence (18) was applied to establish a modified grey GM $(0,1 \mid \sin )$ model of oscillation sequences:

$$
\begin{aligned}
& x^{(0)}(t) \\
& =227.4067 \\
& \times \exp \left(\frac{0.2479 \cos ((11 \pi / 12) t)+0.1189 \sin ((11 \pi / 12) t)}{\pi}\right. \\
& \quad+0.0180 t) \quad t=1,2,3, \ldots
\end{aligned}
$$


TABLE 2: The predicted values by different models and their relative error.

\begin{tabular}{|c|c|c|c|c|c|}
\hline \multirow{2}{*}{ Time ID } & \multirow{2}{*}{ Actual value } & \multicolumn{2}{|c|}{ GM $(0,1 \mid \sin )$ model } & \multicolumn{2}{|c|}{ Modified GM $(0,1 \mid \sin )$ model } \\
\hline & & Predicted values & Relative error (\%) & Predicted values & Relative error (\%) \\
\hline 1 & 264.3 & 264.3 & 0.00 & 264.3 & 0.00 \\
\hline 2 & 327.1 & 295.4 & -9.69 & 302.1 & -7.64 \\
\hline 3 & 317.6 & 280.8 & -11.59 & 284.4 & -10.45 \\
\hline 4 & 286.2 & 293.6 & 2.59 & 300.1 & 4.86 \\
\hline 5 & 292.2 & 300.4 & 2.81 & 308.5 & 5.58 \\
\hline 6 & 300.5 & 291.4 & -3.03 & 297.6 & -0.97 \\
\hline 7 & 324.4 & 320.1 & -1.33 & 333.1 & 2.68 \\
\hline 8 & 300.1 & 291.6 & -2.83 & 298.0 & -0.70 \\
\hline 9 & 360.0 & 336.8 & -6.44 & 354.3 & -1.58 \\
\hline 10 & 328.5 & 296.6 & -9.71 & 304.4 & -7.34 \\
\hline 11 & 368.9 & 347.8 & -5.72 & 368.5 & -0.11 \\
\hline 12 & 307.6 & 307.6 & 0.00 & 318.2 & 3.45 \\
\hline \multicolumn{2}{|c|}{ MAPE (\%) } & \multicolumn{2}{|c|}{4.64} & \multicolumn{2}{|c|}{3.77} \\
\hline \multicolumn{2}{|c|}{$\beta$} & \multicolumn{2}{|c|}{0.63} & \multicolumn{2}{|c|}{0.68} \\
\hline
\end{tabular}

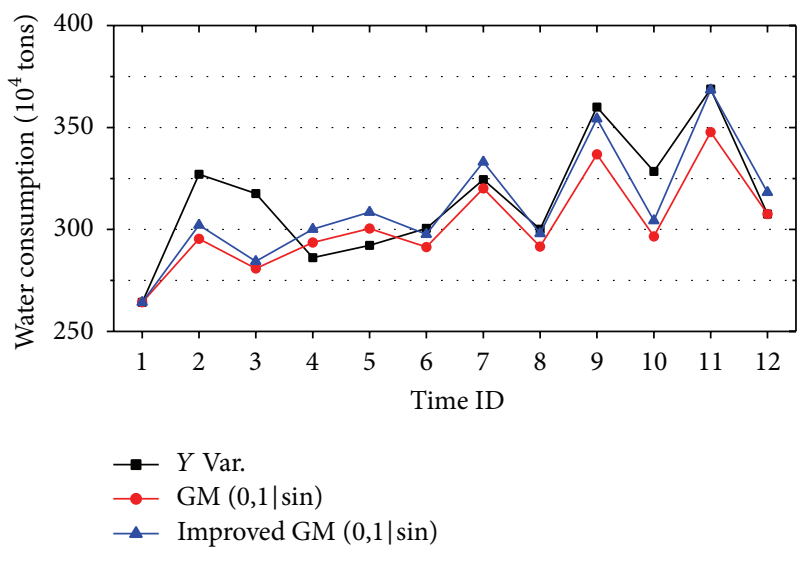

FIgURE 3: The fitting trend using the two models.

The results obtained according to (19) are shown in Table 2.

The comparison of the actual value and the fitting value using the grey GM $(0,1 \mid \sin )$ model of oscillation sequences before and after the modification (Figure 3 ) intuitively displays the fitting effectiveness.

As shown in Table 2, both the GM $(0,1 \mid \sin )$ oscillation model and the modified GM $(0,1 \mid \sin )$ oscillation model with the VWSBO have satisfactory modeling accuracy. The predictive effect of the modified GM $(0,1 \mid \sin )$ oscillation model is relatively superior, with a MAPE of $3.77 \%$ for the fitting. And the comparison between $\beta_{1}$ and $\beta_{2}\left(\left(\beta_{1}=0.63\right)<\left(\beta_{2}=\right.\right.$ $0.68)$ ) further indicates that the improved model fits better to the original sequence. In addition, except for the data point of the third quarter of 2010 (which is abnormal with a large fitting error), the MAPE of this modified model is controlled within $8 \%$ for all of the data, which is superior to the grey GM $(0,1 \mid \sin )$ model of oscillation sequences. The main reason for this superiority is that the modified grey GM $(0,1 \mid \sin )$ model of oscillation sequences was improved by considering the impact of the growth or decay rate of the system data sequence, which is absent from the traditional model.

\section{Conclusions}

For forecasting problems involving a small sample size, uncertainty, and periodic fluctuation, the grey GM $(0,1 \mid \sin )$ model of oscillation sequences has unique advantages. However, the system's behavioral data are often susceptible to interference by the affecting system and distorted. The grey GM $(0,1 \mid \sin )$ model of oscillation sequences often ignores the data distortion caused by an interfering disturbance. For the problem of uneven changes in water consumption in hospitals, this study introduced the VWSBO to weaken the interfering disturbance on the data sequence and to establish a grey $\operatorname{GM}(0,1 \mid \sin )$ model of oscillation sequences with the VWSBO. Based on the real-life data, the MAPE of the fitting using our modified model is $3.77 \%$, which is significantly superior to the traditional grey GM $(0,1 \mid \sin )$ model of oscillation sequences. This model provides a new approach to predict water consumption for these hospitals.

In future studies, the model can be further optimized and applied to other industries. Moreover, a visual decisionsupport system for water resource management can be constructed in combination with geographic information system (GIS) technology to improve the efficiency and level of water resource management and planning. In addition, we have been trying to use Monte Carlo method to study the uncertainty in water resources prediction in a reduced time scale and construct a dynamic model of water quotas in the hospital industry by using the principles of partial least squares (PLS).

\section{Conflict of Interests}

The authors declare that there is no conflict of interests regarding the publication of this paper. 


\section{Acknowledgments}

The work presented in this paper was supported by the National Natural Science Foundation of China (nos. 41071104, 41171319, and 40901214), the Youth Chenguang Project of Science and Technology of Wuhan City of China (no. 200950431203), and Hubei Province Science Fund for Distinguished Young Scholars (2009CAD015).

\section{References}

[1] W. Shuai and S. Yuefeng, "Urban water consumption prediction based on a partial least-square model coupled with stepwise regression," Journal of Safety and Environment, vol. 12, pp. 170$173,2012$.

[2] Z. Wei, Z. Zhihong, and W. Huiwen, "Prediction model of urban daily water consumption and its application," System Engineering, vol. 28, pp. 93-97, 2010.

[3] X. Ping, Z. Meng, Z. Zhi, and Z. Nan, "Period-divied predictive model of urban hourly water consumption based on bp neural network," Journal of Central South University: Science and Technology, vol. 43, pp. 3320-3324, 2012.

[4] F. K. Odan and L. F. R. Reis, "Hybrid water demand forecasting model associating artificial neural network with fourier series," Journal of Water Resources Planning and Management, vol. 138, no. 3, pp. 245-256, 2012.

[5] Z. Ming-Chang and C. Jyh-Horng, "A novel nonlinear forecasting model for output of bike industry by grey model and taguchi-differential evolution algorithm," African Journal of Business Management, vol. 5, pp. 4945-4954, 2011.

[6] D.-C. Li, C.-J. Chang, C.-C. Chen, and W.-C. Chen, "Forecasting short-term electricity consumption using the adaptive greybased approach-an Asian case," Omega, vol. 40, no. 6, pp. 767773, 2012.

[7] S.-L. Ou, "Forecasting agricultural output with an improved grey forecasting model based on the genetic algorithm," Computers and Electronics in Agriculture, vol. 85, pp. 33-39, 2012.

[8] Z. Fangjin, C. Xingcheng, W. Jun, Y. Jiyang, and G. Huirong, "Application of grey model method to vacuum preloading settlement and consolidation degree prediction," Journal of Jiangxi University of Science and Technology, vol. 33, pp. 28-32, 2012.

[9] R. Ye, X. Lu, and H. Liu, "Local tomography based on grey model," Neurocomputing, vol. 101, pp. 10-17, 2013.

[10] X. Yang, D. Kong, and B. Yu, "Prediction analysis for typhoid and paratyphoid fever with $\mathrm{Gm}(1,1)$ grey model in Wuhan," Journal of Public Health and Preventive Medicine, vol. 23, pp. 46, 2012.

[11] K. Hsu, "Using $\mathrm{GM}(1, \mathrm{~N})$ to assess the effects of economic variables on bank failure," Journal of Grey System, vol. 23, no. 4, pp. 355-368, 2011.

[12] Q.-F. Li, Y.-G. Dang, and Z.-X. Wang, "Analysis of the regional coordination development systems based on Gra and Gm(1,n)," Journal of Grey System, vol. 24, pp. 95-100, 2012.

[13] C.-M. Hsiao and S.-M. Wang, "The analysis of the trend of foreign students coming to taiwan for higher education and the marketing strategy," Journal of Grey System, vol. 21, no. 4, pp. 387-394, 2009.

[14] M. Fang, G. Zhang, L. Zhao, and X. Lin, "Forecast of water consumption of Hefei based on $\mathrm{Gm}(1,1)$ model," Journal of Xian
University of Architecture \& Technology: Natural Science Edition, vol. 42, pp. 862-866, 2009.

[15] S. Shu, G. Xiang, W. He, C. Wu, M. Zhao, and Y. Yuan, "Application of $\operatorname{GM}(1,1)$ in long-term urban water demand forecast," Journal of Harbin Institute of Technology, vol. 41, no. 2, pp. 85-87, 2009.

[16] Y. Gan, X. Chen, J. Fu, and X. Yuan, "Prediction of urban domestic water demand based on $\mathrm{Gm}(1,1)$ model," Water Resources and Power, vol. 30, pp. 40-42, 2012.

[17] X. Xiao, Grey Forecasting and Decision-Making Methods, Science Press, Beijing, China, 2013.

[18] S. Liu, "The three axioms of buffer operator and their application," The Journal of Grey System, vol. 3, no. 1, pp. 39-48, 1991.

[19] S. Liu, "The trap in the prediction of a shock disturbed system and the buffer operator," Journal of Huazhong University of Science \& Technology, vol. 25, pp. 25-27, 1997.

[20] Z. Wang, Y. Dang, and S. Liu, "Study on buffer operators with variable weights and their effect strength to original sequence," Control and Decision, vol. 24, no. 8, pp. 1218-1222, 2009.

[21] Y. Gao, D. Zhou, C. Liu, and L. Zhang, "Constructing methods of new buffer operators with variable weights and their inner link," System Engineering Theory \& Practice, vol. 33, no. 2, pp. 489-497, 2013. 


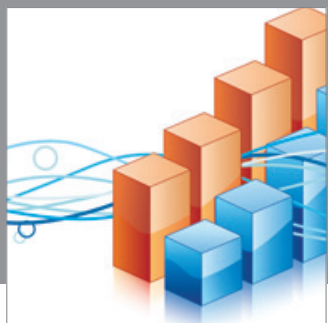

Advances in

Operations Research

mansans

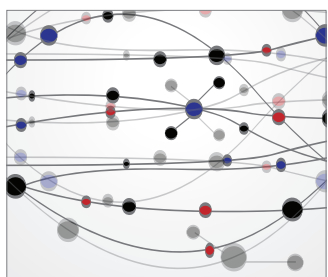

The Scientific World Journal
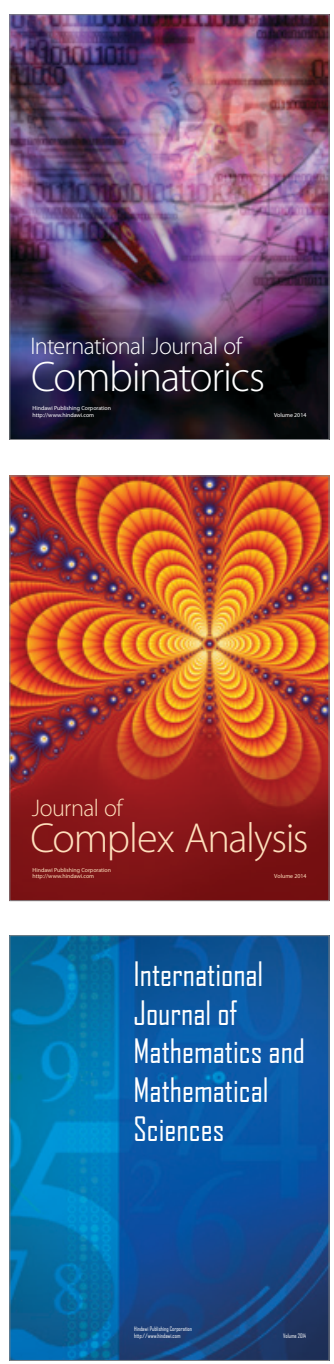
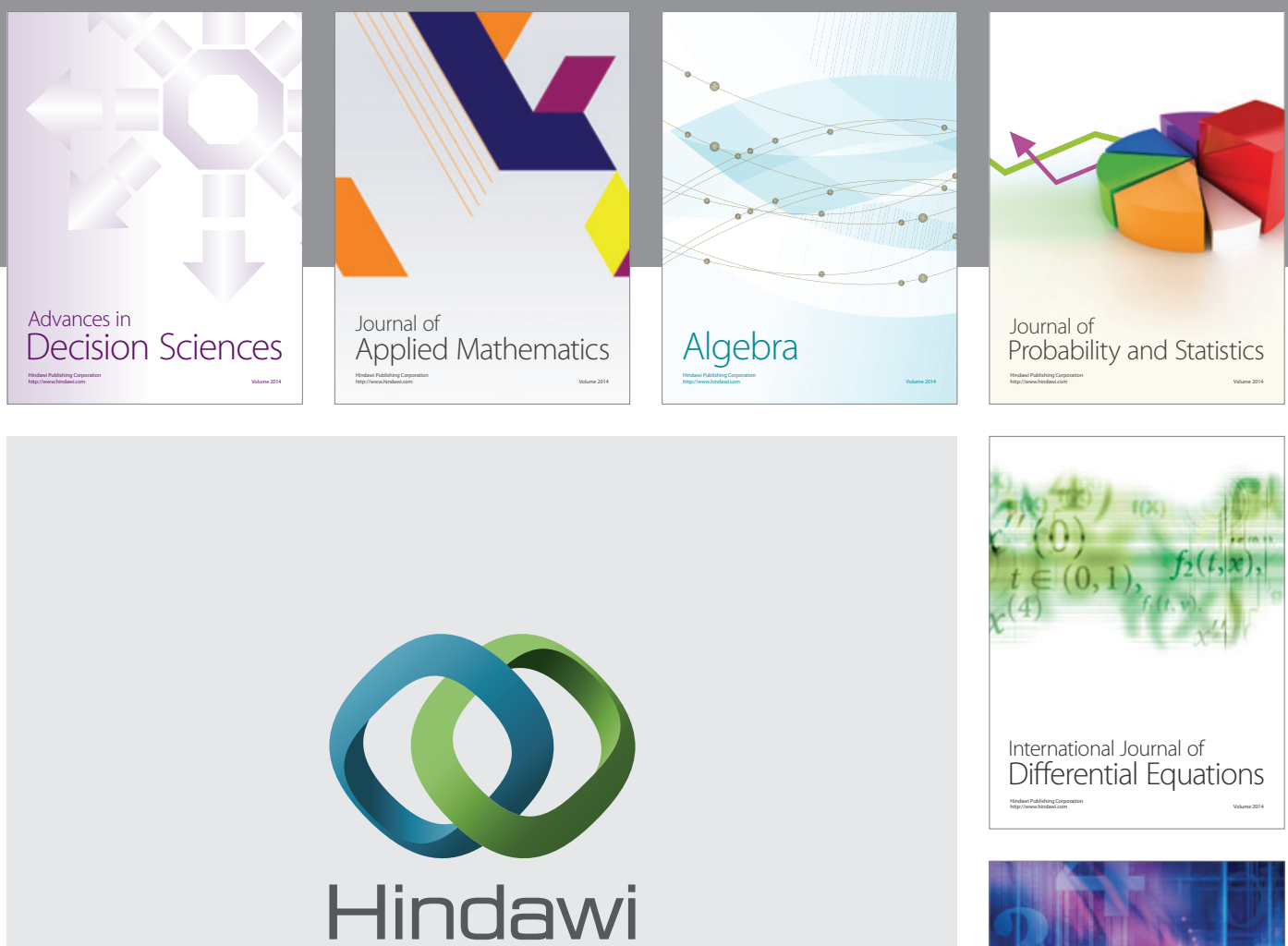

Submit your manuscripts at http://www.hindawi.com
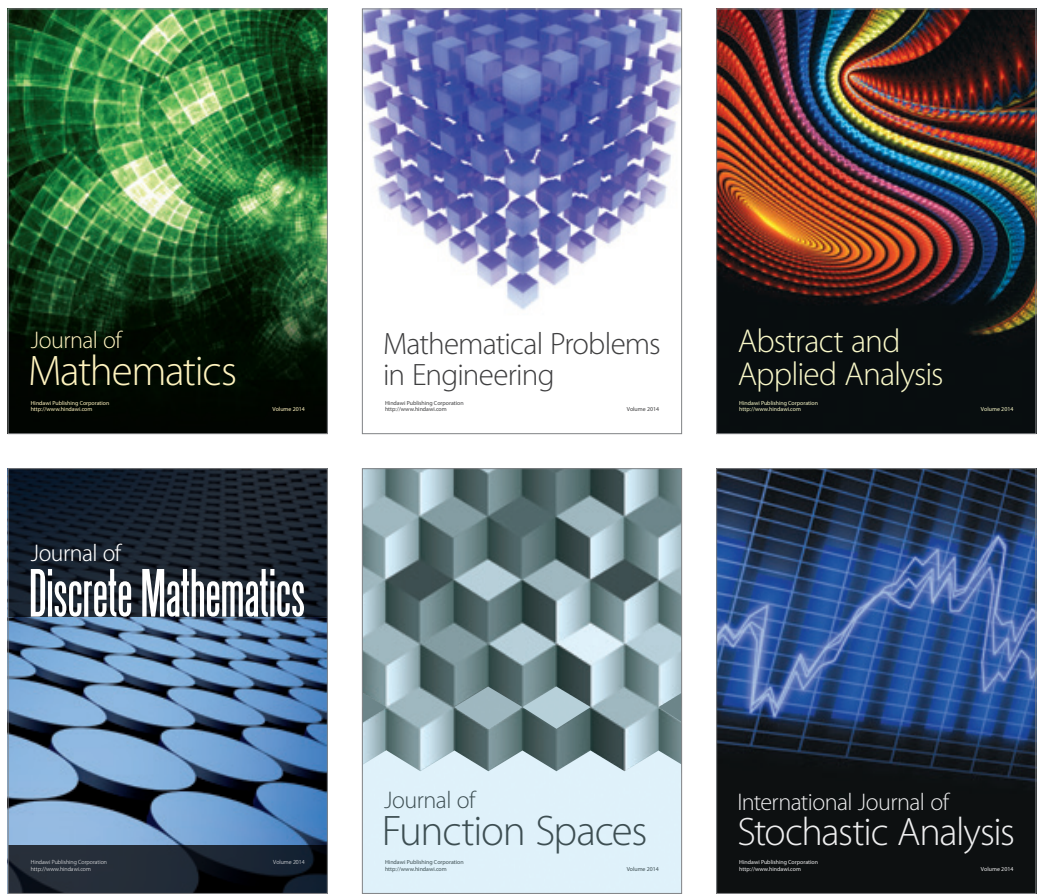

Journal of

Function Spaces

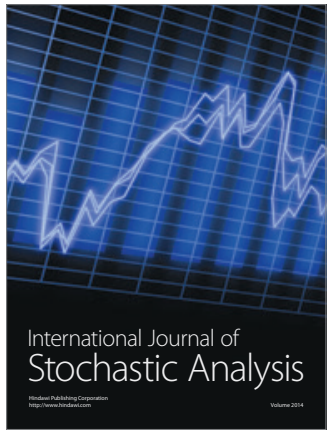

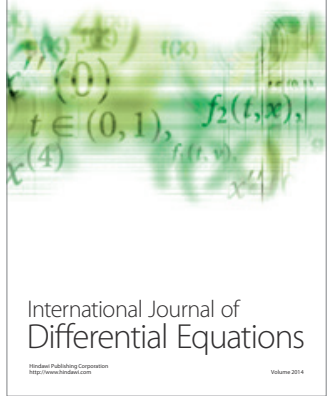
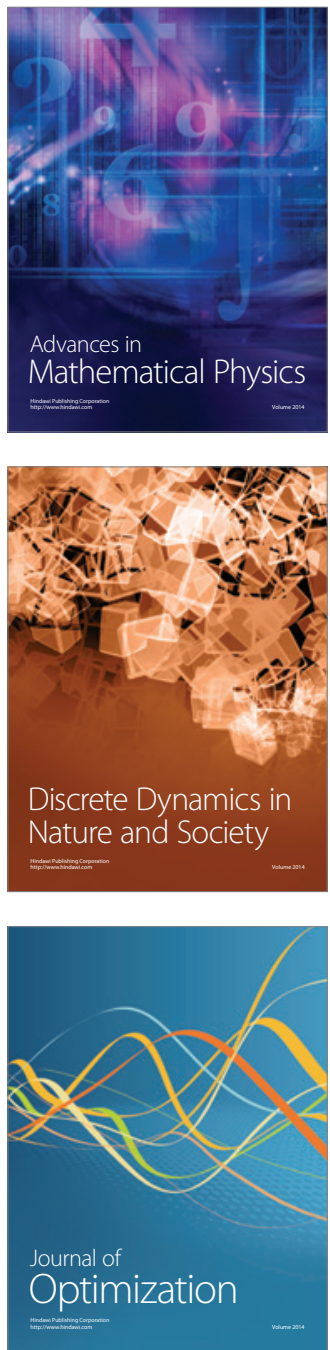